\section{FOUNDATIONS \\ A ADVANCES}

ISSN 2053-2733

Keywords: rotation matrices; irreducible representations; fast rotation function

\section{Accurate computation of the rotation matrices. Errata}

Jorge Navaza*

ER 180 du CNRS, Centre Pharmaceutique, 92290 Chatenay Malabry, France. * Correspondence e-mail: jorge.navaza.estevez@gmail.com

Two equations in the article by Navaza [Acta Cryst. (1990), A46, 619-620] are corrected.

Equations (3) and (5) in Navaza (1990) are corrected. Equation (3) should read

$$
\begin{aligned}
d_{m j}^{j}(\beta)= & \{(2 j) ! /[(j+m) !(j-m) !]\}^{1 / 2} \\
& \times \sin (\beta / 2)^{j-m} \cos (\beta / 2)^{j+m}
\end{aligned}
$$

and equation (5) should read

$$
\begin{aligned}
& {[(j-n+1)(j+n)]^{1 / 2} d_{m, n-1}^{j}(\beta)} \\
& \quad+[(j+n+1)(j-n)]^{1 / 2} d_{m, n+1}^{j}(\beta) \\
& \quad+2(m-n \cos \beta) \sin ^{-1}(\beta) d_{m, n}^{j}(\beta)=0 .
\end{aligned}
$$

\section{References}

Navaza, J. (1990). Acta Cryst. A46, 619-620.

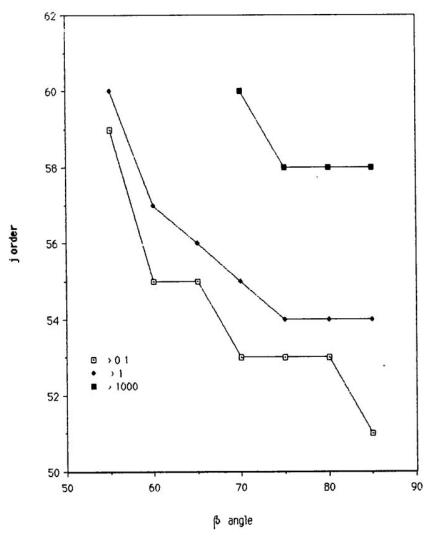

C) 2020 International Union of Crystallography 